Proceedings of the Operational Research Society Simulation Workshop 2021 (SW21)

M. Fakhimi, D. Robertson, and T. Boness, eds.

DOI: https://doi.org/10.36819/SW21.020

\title{
STRATEGIC DECISION MAKING FOR THE NORTH WEST AIR AMBULANCE CHARITY USING DISCRETE EVENT SIMULATION
}

\author{
Dr Lucy E. Morgan \\ Lancaster University \\ Lancaster University Management School \\ 1.e.morgan@lancaster.ac.uk
}

\author{
Dr. Roger J. Brooks \\ Lancaster University \\ Lancaster University Management School \\ roger.brooks@lancaster.ac.uk
}

\begin{abstract}
This paper is a preliminary investigation of the provision of enhanced emergency health care at night by North West Air Ambulance (NWAA). A discrete event simulation model of the current NWAA operational system is presented. This model is used to investigate the provision of NWAA services by an air ambulance vs. a rapid response vehicle. More widely, this model will be used within the scope of investigating what NWAA describe to be their 'optimal service hours'.
\end{abstract}

Keywords: Discrete Event Simulation, Air Ambulance, Helicopter Emergency Medical Service.

\section{INTRODUCTION}

North West Air Ambulance (NWAA) are a charity funded organisation that provide enhanced healthcare interventions throughout the Northwest in the areas of Greater Manchester, Lancashire and Cumbria. NWAA provide advanced medical expertise and equipment that can be utilised at the scene of an emergency. They can also facilitate conveyance of patients to hospital when required. At present the charity has six vehicle assets, three air ambulances (helicopters) and three rapid response vehicles (RRVs), and attend over 2,000 missions each year. NWAA have three advanced healthcare teams and two base sites; two of the healthcare teams are based at Barton near Manchester and one at Blackpool. All team members have received advanced training for the types of missions they are likely to attend with NWAA. The teams are made up of specialist paramedics and/ or a highly trained doctor (consultant or anaesthetist). Each team can be assigned to a single helicopter or RRV; therefore there is a maximum of three NWAA assets in use at any one time. See Table 1 for a comprehensive list of the NWAA assets, including the asset call signs and air ambulance and RRV pairings.

In this paper we present the initial results of investigating the strategic working hours of NWAA. The problems faced by NWAA concern what they describe as 'optimal working hours' i.e. what resources to provide at which times of day. In this paper we describe a simulation model developed in WITNESS to

Table 1: A breakdown of the NWAA assets, their base locations and healthcare teams.

\begin{tabular}{|l|l|l|}
\hline Asset (call sign) & Base location & Healthcare team \\
\hline \hline $\begin{array}{l}\text { Helicopter (H72) } \\
\text { RRV (HX01) }\end{array}$ & Barton & Consultant + Paramedic \\
\hline $\begin{array}{l}\text { Helicopter (H75) } \\
\text { RRV (HX03) }\end{array}$ & Barton & 2 Paramedics \\
\hline $\begin{array}{l}\text { Helicopter (H08) } \\
\text { RRV (HX02) }\end{array}$ & Blackpool & 2 Paramedics \\
\hline
\end{tabular}




\section{Morgan and Brooks}

evaluate a range of service hour options and then describe its use to consider one particular issue, providing single resources outside of daylight hours. Currently NWAA complete missions during daylight hours only, but have an interest in extending operational hours beyond this if there is evidence of a need for enhanced healthcare provision at night. Note that the operation of air ambulance missions at night comes with increased risk and cost; investment in a new helicopter would be required before NWAA could carry out any air ambulance missions at night. Our interest therefore lies firstly in whether there is the need for NWAA to provide a night time service, and secondly in whether an air ambulance is the most appropriate asset to do this. Could a RRV could provide a comparative service to an air ambulance through the night? No additional investment, other than crew costs, would be required to use a RRV at night.

We state clearly that the work presented in this paper is a work in progress; all results presented are preliminary and may be subject to further investigation.

In Section 2 we briefly discuss the background of air ambulance provision at night and the current literature in the area. In Section 3 we discuss model development for the NWAA operational system including the sources of data used to validate the baseline model and the assumptions/ estimates made during the experiments. In Section 4 we present the results of some preliminary experiments, and in Section 5 we conclude and discuss future experiments.

\section{BACKGROUND AND LITERATURE}

The use of helicopters to provide emergency medical services at night has been a topic of interest for some time in the UK. In North America and continental Europe night flying air ambulances are already common McQueen et al. (2015), but such missions come with additional costs and risk. All night time flights in the UK must adhere to the Civil Aviation Safety Directive (CAA 2019).

To date retrospective studies have been popular for identifying the number of incidents an air ambulance may have attended during the night over a given period. Lyon et al. (2015) use a retrospective analysis of trauma patients as part of their investigation into the need for a UK helicopter emergency medical service by night in Kent, Surrey and Sussex (KSS). Their analysis included five independent Helicopter Emergency Medical Service (HEMS) clinicians who were asked to identify which patients met HEMS activation criteria. Within this investigation they also completed a prospective study in which a HEMS dispatch paramedic was present in the ambulance dispatch centre during the night to identify HEMS activation cases. This study was used to motivate the need for a night time air ambulance service in KSS. Curtis et al. (2017) followed up on this work by looking at the implementation of an emergency medical night time service in KSS over a two year period. This paper sought to compare the actual need for the HEMS night time service to the previously estimated need. A number of interesting results arose from this study including the increased number of conveyances of patients to hospital, the greater severity of injury of patients and the introduction of a mission planning phase into mission cycle time during the night. Note that this practical study also reported that significant delays were encountered when the enhanced care team had to respond via RRV during nights when weather meant the air ambulance could not fly.

McQueen et al. (2015) also use a retrospective study to investigate whether helicopters are the answer to responding to major trauma incidents in the West Midlands (WM). Cases likely to meet helicopter activation criterion were identified from historical data by considering injury severity, enhanced care team activations and location. Unlike in KSS the WM already provided emergency care response at night using a fast response vehicle; during the day this team utilised an air ambulance. In the WM a number of voluntary care teams also exist throughout the region to provide emergency health provisions, and before the study the WM were already said to provide good emergency healthcare provision during the night. This may be a result of the good road networks in the region and the volunteer services. Using evidence from the retrospective study McQueen et al. (2015) found that there was little evidence to suggest the need for an air ambulance service in the WM during night.

These sources highlight the importance of emergency health care services at night whether that provision is delivered by an air ambulance or not. It is not clear which characteristics lead to the need for night 
Table 2: A description of the possible NWAA mission results.

\begin{tabular}{|l|l|}
\hline Job Result & Description \\
\hline SD & NWAA have accepted the job, but are stood down at take off, en route or at the scene. \\
\hline TR & NWAA treat a patient/ patients at scene. \\
\hline CO & NWAA convey a patient or follow the ground escort to hospital. \\
\hline Missed & The NWAA asset required for mission is unavailable, and the job is missed. \\
\hline
\end{tabular}

time air ambulance services; this may depend on the characteristics of the region including the current healthcare provisions. Another point of interest is how both studies only considered trauma patients to motivate the need for air ambulance services at night. Whilst trauma patients make up a large part of the number of patients NWAA attend to, their dispatch criteria is not exclusive to trauma patients.

\section{THE MODEL}

Before considering any changes to NWAA operations we first required an understanding of the current operational system and a model that accurately reflected it. Access was provided to the NWAA owned database, HEMSBase, by NWAA. The HEMSBase provided a great amount of detail on each mission carried out by NWAA including exact times for take off, mission cycle time and the mission result. To gain understanding of which entries were of greatest importance we liaised with managers and medical staff from NWAA. After these discussions and some initial data exploration we developed a simple conceptual model of the job process within the NWAA system, see Figure 1. The NWAA job process comprises of: a job arriving, when occurs when an emergency call has been flagged as requiring NWAA assistance; a job being allocated to an appropriate asset; mission time which can include travel time and treatment and/or conveyance if required, and the job leaving the NWAA system; which can describe NWAAs part in the mission being complete or NWAA rejecting/ missing a job.

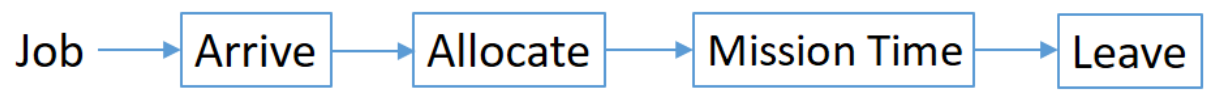

Figure 1: A conceptual model of the job process.

On further consideration of the HEMSBase we were able to pick out the input models we would use to drive our simulation of the NWAA system. We first considered the distribution of the jobs over different days and found that whilst the behaviour on weekdays was reasonably constant the difference between weekdays and weekends was significant. For the remainder of this paper we shall focus on weekdays alone. Further investigation is required to draw conclusions about weekends although many of the decisions we made about the input models still hold.

For weekdays simple analysis confirmed that whilst the arrival profile was not significantly different, both the the proportion of arrivals and the mission cycle times were significantly different for each asset. We therefore estimated a single arrival profile for all six assets and estimated the proportion of jobs to assign to each asset using the number of jobs completed per asset during periods when all assets were on duty. The arrival profile was approximated by a Poisson process with a piecewise constant arrival rate over 30 minute intervals. The mission cycle time distributions for each vehicle were broken down further as analysis of the HEMSBase showed a significant difference between the mission cycle times from missions with different results. The job results recorded in the HEMSBase were: stand down (SD), treatment (TR), conveyance $(\mathrm{CO})$ and missed, see Table 2 for a key to the meaning of the job results. The probability of the job result for each vehicle was inferred directly from the proportion of the results for each asset in the HEMSBase. The job results were found to be independent of vehicle, day of the week and time of day. 


\section{Morgan and Brooks}

When fitting the input models we considered entries from the HEMS database between $28^{\text {th }}$ March 2018 and $30^{\text {th }}$ July 2019. In this period NWAA saw 3,426 total jobs and treated 1,796 patients.

\subsection{Inferring First Preferences}

Although the information in the HEMSBase was very detailed it did not list the first preference vehicle, air ambulance or RRV, for each mission. Whilst the database did record which vehicle actually completed the mission we had no data on instances when the preferred vehicle was busy and another vehicle was sent in its place. We therefore had to infer the preference for each vehicle and the second (and sometimes third) preference if that asset was unavailable so we could mimic the correct job allocation behaviour within our DES model. Note that we assume that missions with first preference of a helicopter will only be reallocated to other helicopters and not RRVs and vice versa. To estimate first preferences we used two distinct but complementary approaches. The first approach was data driven; first preferences were inferred by considering the proportion of times each vehicle was allocated to a mission at times when all three healthcare teams were free. Intuitively if all assets are free then the chosen asset will have been first preference. The arrival rate when everything is available will also be a "true" arrival rate with no rejected jobs (this deals with the general modelling problem of estimating an arrival rate when some customers do not enter the system when it is busy). Second preferences were calculated similarly from the missions observed when a single asset, say the doctor helicopter (H72), was busy. At times when H72 is busy it cannot be allocated to missions; some of the missions that arrive during the times $\mathrm{H} 72$ is busy will have had first preference $\mathrm{H} 72$, but not all. To estimate the second preference proportions for H72, say the probability H75 was the second preference, we first use the estimated first preference proportions for all the other assets and remove these jobs and then look at the proportion of remaining jobs that were done by $\mathrm{H} 75$. This process was repeated for all assets. Third preferences were estimated similarly, but we noticed that in the HEMSBase when two assets were busy a number of jobs were rejected. We therefore also allowed for a small probability of rejecting a mission when two of the three assets were busy.

Our second approach to inferring the first choice preferences considered the utilisation rate, $U_{i}$, of each asset. The basis of this method is to recognise that the rate, $J_{i}$, at which an asset $i$ undertakes a job is a linear function of the total arrival rate of jobs $\lambda$, asset utilisation $U_{i}$ and the asset preference probabilities. Let $\pi_{i}$ denote the first preference probability for vehicle $i$ and $\pi_{k i}$ denote the probability asset $i$ is the second preference when asset $k$ was the first, then

$$
J_{i}=\lambda \pi_{i}\left(1-U_{i}\right)+\sum_{k \neq i} \lambda \pi_{k} U_{k} \pi_{k i}
$$

Therefore, since the utilisation rate, $U_{i}$, is just the rate of jobs undertaken, $J_{i}$, multiplied by the mean service time of the asset, denoted $\tau_{i}$, for each asset we have

$$
U_{i}=J_{i} \tau_{i}=\left(\lambda \pi_{i}\left(1-U_{i}\right)+\sum_{k \neq i} \lambda \pi_{k} U_{k} \pi_{k i}\right) \tau_{i}
$$

This can be expressed in matrix format for the three helicopters as follows

$$
\lambda\left(\begin{array}{ccc}
\left(1-U_{H 72}\right) \tau_{H 72} & U_{H 75} \pi_{H 75 H 72} \tau_{H 72} & U_{H 08} \pi_{H 08 H 72} \tau_{H 72} \\
U_{H 72} \pi_{H 72 H 75} \tau_{H 75} & \left(1-U_{H 75}\right) \tau_{H 75} & U_{H 08} \pi_{H 08 H 75} \tau_{H 75} \\
U_{H 72} \pi_{H 72 H 08} \tau_{H 08} & U_{H 75} \pi_{H 75 H 08} \tau_{H 08} & \left(1-U_{H 08}\right) \tau_{H 08}
\end{array}\right)\left[\begin{array}{c}
\pi_{H 72} \\
\pi_{H 75} \\
\pi_{H 08}
\end{array}\right]=\left[\begin{array}{c}
U_{H 72} \\
U_{H 75} \\
U_{H 08}
\end{array}\right]
$$

Taking the inverse of this matrix gives values for $\pi_{H 72}, \pi_{H 75}$ and $\pi_{H 08}$, but notice that these values are a function of the second preference probabilities, $\pi_{k i}$. In practice the final first preference values were not sensitive to the second preference values, and good estimate values for the first preferences were possible using crude values of the second preferences. This secondary approach gave us the opportunity to double 


\section{Morgan and Brooks}

check the first preferences estimated using the data driven method described above by passing the data driven estimates of the second preferences through the inverse of Equation 1.

Once we had the inferred vehicle preferences for each job this allowed us to correct the percentage of arrivals allocated to each vehicle within the DES model and to build in logic to reroute jobs to other assets if the first preference was found to be busy.

\subsection{Validation}

Before experimenting with our DES model we needed to check that it reflected the real-world behaviour in the current NWAA operational system well; two types of validation exercise were completed to check this. We first preformed a white box validation exercise by discussing the choices behind the model input parameters, model logic and model outputs with representatives from NWAA. After a positive result from this exercise we went on to perform a black box validation exercise by comparing key performance indicators (KPIs) calculated from a single long run of the simulation model to the same KPIs calculated from the missions observed in the HEMBase.

One difference between the simulation model and the true NWAA system is that the simulation model assumes that all assets are available for the same time period each day. In reality, although NWAA planned to start missions at $7 \mathrm{am}$ and end by $7 \mathrm{pm}$ every day, when we looked into the HEMSBase we found that the number of helicopters on duty often fluctuated in the first and last few hours of the day. For validation purposes we therefore focused on the hours where NWAA offer 'full capacity' coverage i.e. all three healthcare teams are on duty at once. This narrowed our validation interval to $11 \mathrm{am}-5 \mathrm{pm}$. A warm up period from 9-11am was used within the simulation model as in reality the system is unlikely to start from empty at $11 \mathrm{am}$. Considering the KPIs of accepted jobs, stand downs and jobs accepted by a helicopter per week Table 3 describes the output of the simulation model compared to the observed missions from the HEMS database. It is clear that although there are differences between the KPIs from the real-world data and the DES model the results are close.

Table 3: Blackbox validation of the DES model for 'full capacity' NWAA system between 11am-5pm.

\begin{tabular}{|l|c|cc|}
\hline KPI & HEMS database & \multicolumn{2}{|c|}{ Model output (CI) } \\
\hline Accepted jobs & 32.89 & 36.46 & $(36.37,36.55)$ \\
Stand downs & 14.81 & 17.66 & $(17.60,17.73)$ \\
Completed by helicopter & 31.43 & 27.91 & $(27.85,27.97)$ \\
\hline
\end{tabular}

Another validation measure was to consider the utilisation of each vehicle, comparing the utilisation profile observed in the simulation to the actual utilisation seen in the HEMS data base. Figure 2 shows the utilisation profile for the three helicopters by call sign (H72 = Barton doctor helicopter, H75 = Barton paramedic and $\mathrm{H} 08=$ Blackpool paramedic) and the busiest of the RRVs (HX01 = Barton doctor RRV). It is clear that the utilisation profiles for each of the vehicles shown in Figure 2 match the utilisation observed in the HEMS database very well.

We are satisfied that we have a valid model of the current operation system for NWAA. We shall now use the DES model to provide some insight into using an air ambulance compared to a RRV at night.

\section{PRELIMINARY EXPERIMENTS}

Recall that our aim is to investigate which assets NWAA should provide at which times of the day. Towards this we will now present our preliminary results for comparing the use of an air ambulance or a RRV for night-time missions. We again state that the results presented here are preliminary and part of a wider project that is a work in progress. We start by comparing KPIs when both vehicles are assumed to perform the same at night as they do during the day (no change to the mission cycle time distributions). We then consider adding a constant to each mission cycle time to account for the possible increase in mission length 


\section{Morgan and Brooks}
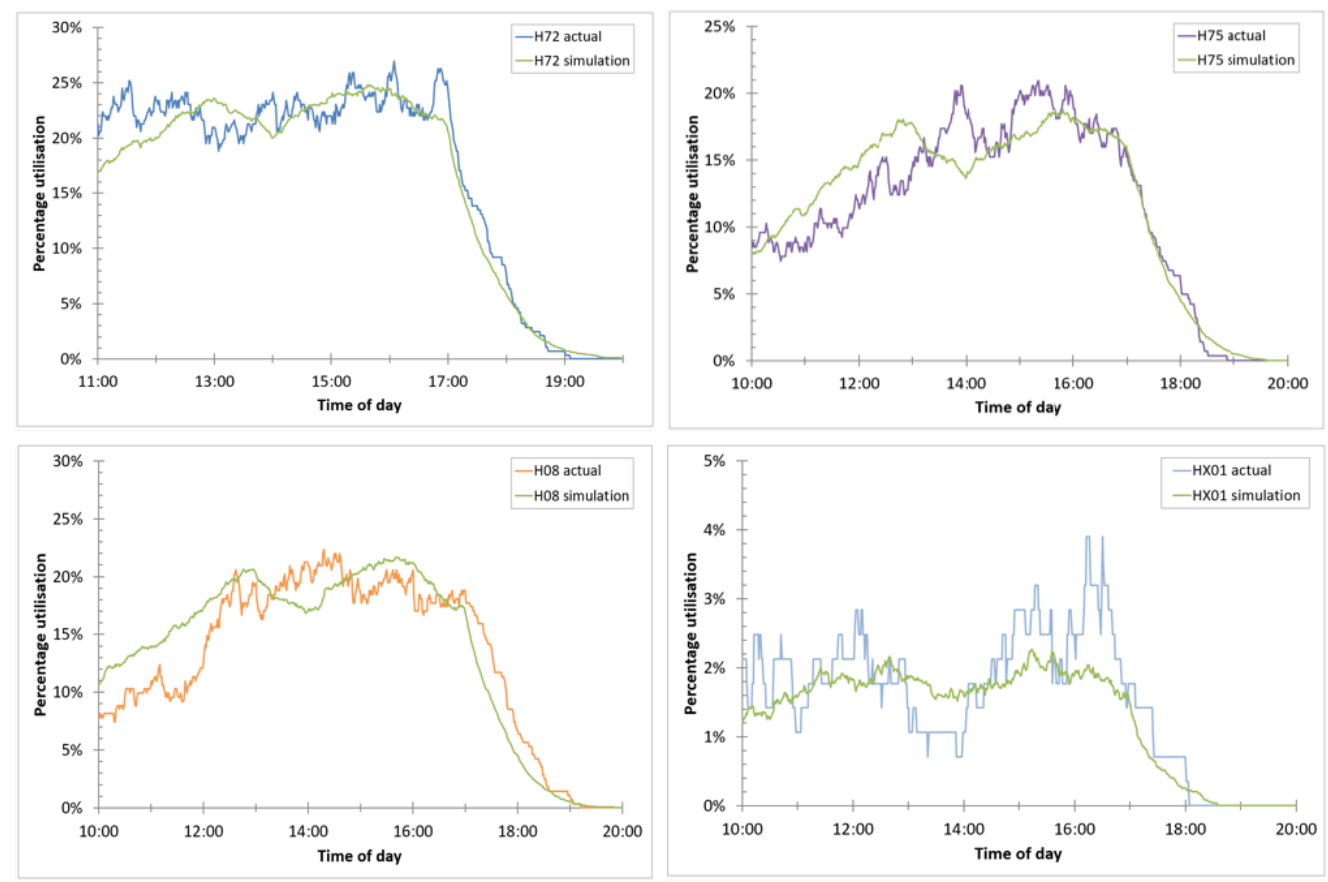

Figure 2: The utilisation profile for the three helicopters $(\mathrm{H} 72=$ Barton doctor helicopter, $\mathrm{H} 75=$ Barton paramedic and H08 = Blackpool paramedic) and HX01 the Barton based RRV with the doctor).

for both vehicles at night. Note that to use an air ambulance during the hours of darkness would require NWAA to invest in a new helicopter with night time flying capabilities; using a RRV would not incur the cost of a new vehicle.

Discussions with NWAA highlighted two possible shift patterns of interest that would incorporate night time missions. The first involved two 10 hour shifts per day (7am-5pm and $5 \mathrm{pm}-3 \mathrm{am})$ and the second involved two 12 hour shifts per day (7am-7pm and $7 \mathrm{pm}-7 \mathrm{am})$. In this experiment our interest lies in the operational performance of NWAA during the night shift we therefore only report our KPIs for missions undertaken during the night. For the purpose of our experiments in the second shift of the day, we assume that a single asset is on duty; this could be either an air ambulance or RRV. We also assume that there is always an asset available at the start of the night shift i.e. the night shift starts from empty with no warm up required. This is a reasonable assumption as we expect the utilisation of the assets to be low at the end of the day, and the health care team would need to swap over between the day and night shift.

Since there were no observations in the HEMSBase on arrivals to the NWAA system at night we used a second database to infer the number of missions that NWAA would be called out to. This database was provided by the North West Ambulance Service (NWAS). To infer the night time arrival profile we calculated the proportion of jobs completed by NWAA within the NWAS database within the 'full capacity' hours (11am-5pm); it turned out that approximately $5 \%$ of NWAS jobs were completed by NWAA during that period. We therefore assumed the same proportion of the NWAS jobs would be completed by NWAA at night which allowed us to create arrival profiles for the 10 hour shift $(5 \mathrm{pm}-3 \mathrm{am})$ and the 12 hour shift (7pm-7am).

After speaking to experts from NWAA about this assumption it was suggested that the proportion of NWAS jobs completed by NWAA would change during the night due to more serious incidents often occurring in the early morning hours, particularly in densely populated (inner city) areas. We therefore adjusted the estimate arrival profile to take into account the proportion of serious incidents that occurred in the NWAS database at night. This resulted in an arrival profile with an average of 5.5 emergency calls per 


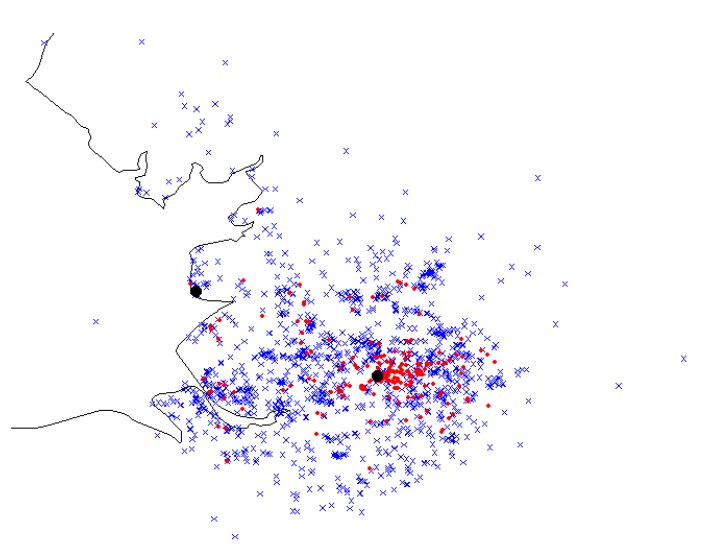

Figure 3: A map of mission locations for the H72 air ambulance (blue) and HX01 RRV (red) during the day. The Barton and Blackpool bases are also marked (black).

night for the 10 hour night shift and 4.8 emergency calls per night for the 12 hour night shift. We assume that as there is only one asset available in the evening all arrivals would be allocated to this resource during the night shift. If that resource is busy then the job is missed. We also assume that the healthcare team available to the asset throughout the night is the highly skilled doctor/consultant and paramedic paring. The NWAA asset on the night shift is therefore equivalent to either the Barton doctor helicopter (call sign: H72) or the Barton doctor RRV (call sign: HX01).

Estimation of the effect of night-time flights on the mission cycle-time distributions used within our model were harder to infer. Discussions with NWAA highlighted that helicopter flights at night would take longer to prepare for take off due to additional checks/ planning being required, and would also take longer to land at scene due to the larger landing zone required in the hours of darkness. McQueen et al. (2015) also discuss the problem of many hospital helipads in the UK lacking the infrastructure for night time landings which again would add additional time on to any conveyance mission. For these reasons we suspected that night time mission cycle times would need to be increased, but we had no data to infer by how much we should increase them. Using the current mission cycle time distributions would clearly give an optimistic view of the air ambulances performance as these distributions were fitted from missions that occurred during the day.

We also had little information on the expected mission cycle time of a RRV during the night. NWAA performed a short experiment in January 2019 that saw a their fast response land vehicle respond to incidents during the night, but this experiment coincided with filming for a BBC series and only few missions were accepted. We therefore did not use the data from this experiment to fit our mission cycle time distributions. Inferring the mission cycle time distributions having no data is a difficult problem. Assuming both the air ambulances and RRVs would be based at the Barton site at night we started by comparing the geographical locations of the missions that $\mathrm{H} 72$ and HX01, the vehicles with doctors, completed during the day, see the red points in Figure 3. It is clear from Figure 3 that the RRV tends to attend missions close to the Barton base. Using the current mission cycle time distribution for HX01 should therefore be thought of as being overly optimistic as the travel time is likely to be higher at night when the RRV has to cover a wider area.

Despite both asset mission cycle time distributions being overly optimistic the natural preliminary experiment to perform was a comparison of the two assets at night assuming they could perform as they did during the day, see Table 4. Note that for this experiment we set the proportion of stand down, treatment and convey results to be the same for each asset as the job result is likely to be the property of a mission not the type of vehicle; both assets therefore saw the same proportion of job results. The job result proportions were inferred from the day time missions for the $\mathrm{H} 72$ helicopter. This preliminary experiment was replicated $n=50$ times. In Table 4 we report the mean result of the replications along with a $95 \%$ confidence interval. 


\section{Morgan and Brooks}

Table 4: Comparing KPIs for night time missions undertaken by either a single helicopter or a single RRV assuming night and day time mission cycle time distributions are the same. The KPIs reported are the average of 50 simulation replications; they are reported with $95 \%$ confidence intervals.

\begin{tabular}{|l||c|c||c|c|}
\hline \multicolumn{1}{|c||}{} & \multicolumn{2}{c||}{ 10 hour shift } & \multicolumn{2}{c|}{12 hour shift } \\
\hline & Helicopter & RRV & Helicopter & RRV \\
\hline \hline Total jobs / wk & $38.6(38.44,38.75)$ & $38.0(37.90,38.11)$ & $34.0(33.87,34.17)$ & $33.2(33.15,33.24)$ \\
\hline Missed jobs \% & 30.5 & 28.0 & 25.1 & 22.5 \\
Missed jobs / wk & $11.8(11.67,11.84)$ & $10.6(10.54,10.65)$ & $8.5(8.46,8.62)$ & $7.46(7.40,7.51)$ \\
\hline Accepted jobs \% & 69.5 & 72.0 & 7.49 & 77.5 \\
Accepted jobs / wk & $26.8(26.75,26.93)$ & $27.4(27.34,27.48)$ & $25.5(25.38,25.59)$ & $25.7(25.68,25.80)$ \\
\hline Stand down \% & 48.1 & 48.2 & 48.1 & 47.6 \\
Stand down / wk & $12.9(12.84,12.97)$ & $12.9(12.87,12.98)$ & $12.25(12.19,12.32)$ & $12.26(12.20,12.32)$ \\
\hline
\end{tabular}

Table 4 illustrates that if both assets were to perform as they do during the day shift then they would have very similar performance during the night. Note that during the night shift we attempt to allocate all of the missions to a single asset. The number of missed jobs is therefore likely to be higher than in the day when three assets are available. This is reflected in the results, even with both assets performing as they do in the day, which is optimistic as discussed, we see the percentage of missed jobs varies between 22-30\%. This indicates that another resource might be useful during the night shift. The experiment also highlights that the chosen shift pattern matters. In Table 4 we see that the 12 hour shift has fewer jobs per week on average compared to the 10 hour shift. This occurs because $5-7 \mathrm{pm}$ is a busy period for arrivals and only the 10 hour shift encompasses this period; the 12 hour shift starts at $7 \mathrm{pm}$ at the end of the busy period.

Our second experiment investigates increasing all mission cycle times by a constant to account for the possibility that both vehicles are likely to need take longer to complete a mission during the night. The choice of parameters for the mission cycle time distributions for the air ambulance and RRV for our second experiment stem from our conversations with NWAA. During our meetings NWAA representatives expressed the view that the travel time to an incident for the air ambulance and RRV may be roughly equivalent during the hours of darkness due to the additional time required to plan the mission, for take off and to land the helicopter at scene. Clearly if the mission cycle times were equivalent for the air ambulance and RRV there would be little need to invest in the new helicopter.

Since we cannot quantify how the mission cycle time distributions would change during the night for either vehicle, we instead consider incremental increases in all mission cycle times. This aims to give NWAA an idea of how the assets might perform if they were a certain amount slower on each mission than the $\mathrm{H} 72$ helicopter during the day. Let us denote the mean mission cycle time of the air ambulance during the day by $\mu$ (for $\mathrm{H} 72 \mu \approx 49$ mins). Our next experiment considers our KPIs when all mission cycle times of $\mathrm{H} 72$ are increased by a constant of either $10 \% \mu, 50 \% \mu$ or $100 \% \mu$ i.e. all missions were either 4.9, 24.5 or 49 minutes longer.

We chose to increase all mission times by a constant rather than a percentage as the type of vehicle and time of day are only likely to affect the travel time to scene and not other components of the mission cycle time; the addition of a constant amount onto all mission cycle times therefore seemed more appropriate. This experiment will allow us see how sensitive our KPIs are to the mission cycle time distributions. It will also allow us to judge whether the air/ RRV provide a reasonable service even if they have much longer mission cycle times. Future work is needed to better estimate the mission cycle times of both assets.

The results of the experiments for the two night time shift patterns are displayed in Tables 5 and 6 . All experiments were replicated $n=50$ times, the results in table 5 and 6 are the average of these results reported with $95 \%$ confidence intervals. Common random numbers were used across all experiments. 


\section{Morgan and Brooks}

Table 5: KPIs for the 10 hour night shift missions undertaken by a single RRV assuming all the night mission cycle times are $10 \%, 50 \%$ and $100 \%$ greater than the current mission cycle times of the helicopter. The KPIs reported are the average of 50 simulation replications; they are reported with $95 \%$ confidence intervals.

\begin{tabular}{|c|c|c|c|}
\hline & Helicopter $+10 \% \mu$ & Helicopter $+50 \% \mu$ & Helicopter $+100 \% \mu$ \\
\hline Total jobs / wk & $38.74(38.57,38.91)$ & $38.74(38.57,38.91)$ & $38.74(38.57,38.91)$ \\
\hline Missed jobs \% & 32.5 & 39.6 & 46.4 \\
Missed jobs / wk & $12.59(12.50,12.69)$ & $15.33(15.22,15.44)$ & $17.97(17.85,18.09)$ \\
\hline Accepted jobs \% & 67.5 & 60.4 & 53.6 \\
Accepted jobs / wk & $26.15(26.06,26.23)$ & $23.41(23.33,23.49)$ & $20.77(20.71,20.83)$ \\
\hline Stand down \% & 48.2 & 48.1 & 48.1 \\
Stand down / wk & $12.60(12.54,12.66)$ & $11.27(11.21,11.32)$ & $9.99(9.94,10.05)$ \\
\hline
\end{tabular}

Table 6: KPIs for the 12 hour night shift missions undertaken by a single RRV assuming the night mission cycle times are $10 \%, 50 \%$ and $100 \%$ greater than the current mission cycle times of the helicopter. The KPIs reported are the average of 50 simulation replications; they are reported with $95 \%$ confidence intervals.

\begin{tabular}{|c|c|c|c|}
\hline & Helicopter $+10 \% \mu$ & Helicopter $+50 \% \mu$ & Helicopter $+100 \% \mu$ \\
\hline Total jobs / wk & $34.02(33.87,34.17)$ & $34.02(33.87,34.17)$ & $34.02(33.87,34.17)$ \\
\hline Missed jobs \% & 26.8 & 33.3 & 39.7 \\
Missed jobs / wk & $9.13(9.05,9.21)$ & $11.33(11.24,11.42)$ & $13.51(13.41,13.62)$ \\
\hline Accepted jobs \% & 73.2 & 66.7 & 60.3 \\
Accepted jobs / wk & $24.81(24.72,24.90)$ & $22.69(22.59,22.78)$ & $20.51(20.43,20.59)$ \\
\hline Stand down \% & 48.1 & 48.1 & 48.0 \\
Stand down / wk & $11.92(11.87,11.98)$ & $10.91(10.85,10.97)$ & $9.84(9.78,9.90)$ \\
\hline
\end{tabular}

In Table 5 we report the results of our experiment for the 10 hour night shift. We see that as the mission cycle time increases the percentage of missed jobs increases; this was expected as the single asset has higher utilisation so there is more chance of arrivals finding it busy on entry. We also see that although the number of stand downs is decreasing the percentage stays constant as the mission cycle time increases. In Table 6 we see similar behaviour to Table 5. In general notice that if either asset were to act similarly to $\mathrm{H} 72$ in the day, then the average number of patients assisted i.e. the average number of acceptances minus the average number of stand downs in Table 4, equates to over 13 missions per week. Comparing this to the worst case we investigated where all missions were 49 minutes slower for either asset, we see that NWAA would still assist over 10 people per week on average. This is a positive result. It indicates that the number of people on average that are helped by NWAA per week during the night is not particularly sensitive to the mission cycle time. In truth we do not believe the use of either vehicle would make missions 49 minutes longer. The lack of sensitivity of the KPIs to the mission cycle time indicate that the RRV may be the better choice of vehicle due to the additional cost and risk involved in night time flights. From Tables 5 and 6 it appears that the number of missed jobs is more of an issue than the mission cycle times themselves. This indicates that, if the arrival profile is accurate, a second asset might be useful at night.

Whether an air ambulance or a RRV is the asset of choice, by looking at the arrival profile information alone this investigation has told us that NWAA are likely to see over 30 calls during the night per week. This number is high and we believe motivates the need for some form of emergency health care provision through the night. 


\section{CONCLUSIONS AND FURTHER WORK}

This paper considers the need for an air ambulance vs. a RRV to provide emergency health care through the night in the Northwest region. In conclusion to our preliminary investigation we believe that some form of night time emergency health care provision is required due to the large number of jobs estimated to fall during the night that would meet the HEMS dispatch criteria. We also believe that the more appropriate asset is the RRV at present due to the lack of sensitivity of our KPIs to mission cycle time and the additional risk and cost associated with night time flights. Although at the time of writing the paper the project was ongoing, and further analysis/ experimentation is required to investigate when and where would be most appropriate to provide this service.

Going forward, we also intend to consider the addition of a HEMS RRV to be based roadside within the region during the night shift, as the number of missed jobs was reasonably high even when we were optimistic about the mission cycle time distributions. Consideration of the time of year is also of interest and how might this might effect which asset is of most use as it is likely that time of year effects the night shift more than the day shift. Finally, consideration of how the job result distributions may change during the night should be investigated. Lyon et al. (2015) suggest the patient conveyance needs may be higher at night due to the increased injury severity.

On a different note, research into how the HEMS dispatch criteria might be modified at night, and the knock on effect of this may have on the NWAA provision should be investigated. Both (Lyon et al. 2015) and (McQueen et al. 2015) only consider missions involving trauma patients during the night whereas within our model we took into account all incidents that meet NWAA dispatch criteria during the day.

\section{ACKNOWLEDGMENTS}

We gratefully acknowledge Lanner for allowing us to use the simulation software WITNESS.

\section{REFERENCES}

CAA 2019. “CAP1535: The Skyway Code”. https://publicapps.caa.co.uk/docs/33/CAP1535_Skyway_Code_ V2_PRINT.pdf. Online; accessed 28 October 2019.

Curtis, L., M. Salmon, and R. M. Lyon. 2017. "The impact of helicopter emergency medical service night operations in south East England". Air medical journal 36 (6): 307-310.

Lyon, R. M., J. Vernon, M. Nelson, N. Durge, M. Tunnicliff, L. Curtis, and M. Q. Russell. 2015. "The need for a UK helicopter emergency medical service by night: a prospective, simulation study". Air medical journal 34 (4): 195-198.

McQueen, C., T. Nutbeam, N. Crombie, F. Lecky, T. Lawrence, K. Hathaway, and S. Wheaton. 2015. "Enhanced care team response to incidents involving major trauma at night: Are helicopters the answer?". Injury 46 (7): 1262-1269.

\section{AUTHOR BIOGRAPHIES}

LUCY E. MORGAN is a Development Lecturer in Simulation and Stochastic Modelling in the Department of Management Science at Lancaster University. Her research interests are input uncertainty in simulation models and arrival process modelling. Her e-mail address is 1.e.morgan@lancaster.ac.uk.

ROGER J. BROOKS is a lecturer in the Department of Management Science at Lancaster University. His research areas of interest include conceptual modelling, agent-based simulation, applications of simulation in health, and statistical analysis of sports data and health data. 